\title{
Role of paliperidone extended-release in treatment of schizoaffective disorder
}

This article was published in the following Dove Press journal:

Neuropsychiatric Disease and Treatment

27 September 2010

Number of times this article has been viewed

\section{Carla M Canuso' \\ Ibrahim Turkoz ${ }^{2}$ \\ Dong Jing $\mathrm{Fu}^{3}$ \\ Cynthia A Bossie ${ }^{3}$}

'External Innovation Neuroscience Therapeutic Area, ${ }^{2}$ Biostatistics, Johnson \& Johnson Pharmaceutical Research and Development, LLC, Titusville, NJ, USA; ${ }^{3}$ CNS Medical Affairs, Ortho-McNeil Janssen Scientific Affairs, LLC, Titusville, NJ, USA
Correspondence: Carla M Canuso Senior Director, External Innovation Neuroscience Therapeutic Area, Johnson \& Johnson Pharmaceutical Research and Development,

I 25 Trenton-Harbourton Road,

Titusville, NJ 08560-0200, USA

Tel +l 6097307732

Fax +l 6097303125

Email ccanuso@its.jnj.com
Abstract: Schizoaffective disorder is characterized by the presence of symptoms of both schizophrenia and a major mood disorder. The coexistence of these symptoms can be difficult to manage, and these patients are generally treated with antipsychotics as well as mood stabilizers and/or antidepressants. Additionally, no established treatment guidelines exist for this disorder. This review describes the combined results of two international, double-blind, placebo-controlled clinical studies of paliperidone extended-release (ER), an atypical antipsychotic recently approved in the US for the treatment of schizoaffective disorder. Subjects in these six-week trials were aged 18-65 years, had a diagnosis of schizoaffective disorder based on the Structural Clinical Interview for DSM-IV (Diagnostic and Statistical Manual of Mental Disorders, 4th Edition) Disorders, and were experiencing an acute exacerbation. The subjects from these studies had significant symptomatology as evidenced by a mean (standard deviation) baseline Positive and Negative Syndrome Scale total score of 92.8 (13.0). Based on Young Mania Rating Scale and/or a 21-item Hamilton Rating Scale for Depression score of $\geq 16$ at baseline, $79.5 \%$ and $66.9 \%$ of subjects presented with prominent manic and depressive symptoms, respectively, and $46.4 \%$ presented with mixed symptoms. Approximately half (45\%) of subjects were taking adjunctive mood stabilizers and/or antidepressants. Paliperidone ER was found to be effective in improving psychotic and mood symptoms in these subjects. Paliperidone ER was also effective as monotherapy or adjunctive to mood stabilizers and/or antidepressants for subjects with prominent manic, depressive, or mixed symptoms at baseline. No new tolerability signals were observed in this population. To the best of our awareness, these pooled data provide the largest data set of patients with schizoaffective disorder, and extend our knowledge of disease characteristics and treatment response.

Keywords: paliperidone extended-release, antipsychotic, schizoaffective disorder

\section{Introduction}

The term "schizoaffective", used to describe patients with concurrent schizophrenic and mood symptoms, was first introduced more than 75 years ago. ${ }^{1}$ Diagnosis of schizoaffective disorder requires that patients experience an uninterrupted period of illness with concurrent psychotic and mood symptoms. The psychotic symptoms must be present without any prominent mood symptoms for at least two weeks, yet mood episodes must represent a substantial portion of the total duration of illness. Patients with schizoaffective disorder are further classified as having the bipolar or depressive type (Diagnostic and Statistical Manual of Mental Disorders, 4th Edition [DSM-IV]). Epidemiologic data suggest that schizoaffective disorder is about one third as common as schizophrenia, with a lifetime prevalence of $0.3 \%$, compared with $0.9 \%$ for schizophrenia ${ }^{2}$ and such patients are regularly encountered in psychiatric 
clinic populations. A recent cross-national study found that nearly one third of a globally diverse population of patients with symptoms of psychosis who visited psychiatric settings had schizoaffective disorder. ${ }^{3}$ However, when patients with mental illness are characterized by their need for inpatient psychiatric care, those with a diagnosis of schizophrenia and schizoaffective disorder are almost equally represented, ${ }^{4}$ suggesting that patients with schizoaffective disorder may be more disabled by their illness and consume a disproportionately large share of available inpatient mental health resources.

\section{Characteristics of the schizoaffective disorder population}

Compared with the large number of clinical trials for the treatment of schizophrenia and mood disorders, fewer randomized studies have been conducted for schizoaffective disorder. ${ }^{5-10}$ As such, the demographic and clinical characteristics of the schizoaffective population are not fully understood. Cheniaux and coworkers have suggested that some aspects of schizoaffective disorder, such as the female-to-male gender ratio, age of illness onset, total number of episodes, deficits in cognition, and response to treatment, lie midrange in a spectrum between schizophrenia and mood disorders. ${ }^{11}$ The number of hospitalizations and the number of suicide attempts, however, are higher among those with schizoaffective disorder than in those with either schizophrenia or mood disorder. ${ }^{11}$

A study comparing patients with schizophrenia and patients with schizoaffective disorder (matched for intelligence quotient, education, length of illness, and attention span) found that those with schizoaffective disorder had a less marked negative syndrome and less social avoidance but higher levels of depression and guilt. ${ }^{12}$ Patients with schizoaffective disorder were also more cognitively intact, had shorter inpatient stays, and had a higher prevalence of substance abuse in their families compared with those who had schizophrenia. ${ }^{12}$ A recent analysis of a large Medicaid database found that patients with schizoaffective disorder were significantly younger, more likely to be female, and more likely to be white, Hispanic, or of other non-African American race or ethnicity compared with patients with schizophrenia. ${ }^{13}$ Patients with schizoaffective disorder also were significantly more likely to receive concomitant treatment for substance use or for an anxiety, depressive, bipolar, or other mental disorder, and significantly more likely to be treated with mood stabilizers, antidepressants, and anxiolytics. ${ }^{13}$ In addition to distinctions based on demographic and clinical characteristics, emerging molecular genetic analyses have suggested that schizoaffective disorder may have distinctive genetic risk factors compared with other psychiatric disorders. ${ }^{14-16}$

\section{Treatment strategies}

Coexistent psychotic and prominent affective symptoms can be difficult to address, and management is complicated by the variability in clinical presentations. A combination of medications and psychotherapy is typically required for these patients, yet there are no established treatment guidelines. Atypical antipsychotics have mood-stabilizing properties in addition to antipsychotic efficacy, ${ }^{17-19}$ and thus have the potential to be central in the pharmacologic treatment of schizoaffective disorder. Several small or open-label studies with risperidone in schizoaffective disorder have shown positive results. ${ }^{7,20-22}$ A post hoc analysis of a schizoaffective subpopulation from a comparative study of olanzapine and haloperidol found greater benefits from olanzapine in measures of psychosis and depression, although more improvement was seen in those diagnosed with the bipolar type than the depressive type of illness. ${ }^{23}$ Similarly, two additional post hoc analyses of subpopulations from placebo-controlled studies of ziprasidone and aripiprazole suggested that these agents were efficacious in patients with schizoaffective disorder. ${ }^{24,25}$

To manage the affective and psychotic symptoms, many patients with schizoaffective disorder also receive adjunctive treatment with mood stabilizers and/or antidepressants. ${ }^{26-28}$ A recent Medicaid prescription claims database found that approximately one half of those with schizoaffective disorder were prescribed an antipsychotic plus a mood stabilizer or an antipsychotic plus an antidepressant. ${ }^{13}$ A study of hospitalized patients with schizoaffective disorder in the US determined that antipsychotics and mood stabilizers and/or antidepressants were prescribed together in $87 \%$ of cases. ${ }^{28}$ A recent multinational study found that antipsychotics alone was prescribed for $33.9 \%$ of patients with schizoaffective disorder, whereas $23.1 \%$ were taking an antipsychotic plus a mood stabilizer, $27.7 \%$ were taking an antipsychotic plus an antidepressant, and $7.7 \%$ were taking an antipsychotic plus a mood stabilizer and an antidepressant. ${ }^{3}$ However, the benefits and risks of atypical antipsychotics administered alone or in combination with other treatments have not been established.

\section{Paliperidone extended-release for schizoaffective disorder}

Two large, international, double-blind, placebo-controlled, randomized, six-week studies with paliperidone extended-release 
(ER) were conducted for schizoaffective disorder. ${ }^{29,30}$ Paliperidone ER is the first agent approved by the US Food and Drug Administration and several other countries (Australia, Brazil, New Zealand, and Philippines) to treat patients with schizoaffective disorder, both as monotherapy and as adjunctive therapy with mood stabilizers and/or antidepressants. ${ }^{31}$ In contrast with other antipsychotics, paliperidone ER undergoes limited hepatic metabolism and is therefore less likely to cause hepatically mediated drug-drug interactions in patients receiving multiple medications. ${ }^{32-34}$ This review of pooled data from the combined studies covers the largest data set of patients with schizoaffective disorder from randomized controlled trials to date, and provides insights into the characteristics and treatment responses of this understudied population.

\section{Description of the studies}

Subjects in these studies were aged 18-65 years, with a diagnosis of schizoaffective disorder based on the Structured Clinical Interview for DSM-IV Disorders (SCID). All were experiencing an acute exacerbation of illness as evidenced by a Positive and Negative Syndrome Scale (PANSS) ${ }^{35}$ total score $\geq 60$ and a score $\geq 4$ on at least two of the PANSS items for hostility, excitement, tension, uncooperativeness, and poor impulse control. Prominent mood symptoms were reflected in a score $\geq 16$ on the Young Mania Rating Scale (YMRS) ${ }^{36}$ and/or the 21-item Hamilton Rating Scale for Depression (HAM-D-21). ${ }^{37}$ In the first study, subjects received fixed dosages of paliperidone ER, ie, either the higher dosage (12 mg/day, with the option to reduce to $9 \mathrm{mg} /$ day) or the lower dosage ( $6 \mathrm{mg} /$ day, with the option to reduce to $3 \mathrm{mg} /$ day) ${ }^{29}$ In the second study, paliperidone ER was flexibly dosed, beginning at a dosage of $6 \mathrm{mg} /$ day, with a range of 3 to $12 \mathrm{mg} /$ day ${ }^{30}$ For both studies, any dosing changes had to occur within the first 15 days. Subjects receiving stable regimens of mood stabilizers and/or antidepressants were permitted to continue their ongoing treatment to allow the study of paliperidone ER either as monotherapy or as an adjunct to mood stabilizers and/or antidepressants.

Change in PANSS total score from baseline to endpoint was the primary outcome measure for both studies. Secondary efficacy measures were changes from baseline to endpoint for the Clinical Global Impressions of Severity for Schizoaffective Disorder (CGI-S-SCA), ${ }^{38}$ PANSS factor scores, ${ }^{39}$ a composite response ( $\geq 30 \%$ improvement in PANSS total score and Clinical Global Impressions of Change for Schizoaffective Disorder [CGI-C-SCA] of 1 or 2 [much or very much improved]), and the YMRS and HAM-D-21 scales.
Safety assessments included adverse event reporting, clinical laboratory tests (including prolactin levels), and assessment of movement disorders with the Simpson-Angus Scale (SAS), Barnes Akathisia Scale (BAS), and Abnormal Involuntary Movement Scale (AIMS). ${ }^{40-42}$

Randomization for both studies was stratified by center and by treatment with concomitant medications (with or without mood stabilizers and/or antidepressants). Data from the two schizoaffective studies were integrated (paliperidone ER versus placebo) for all statistical analyses. Efficacy was analyzed using the intent-to-treat analysis set, which included all randomized subjects who received $\geq 1$ dose of study medication and had a baseline and $\geq 1$ postbaseline PANSS assessment. The safety population included randomized subjects who received $\geq 1$ dose of study medication. For each continuous parameter, treatment group differences were analyzed using separate analysis-of-covariance (ANCOVA) models with treatment, protocol, country-within-protocol, concomitant medication stratum as fixed-effect design factors, and baseline score as a covariate. To assess consistency of treatment effect within each subgroup, separate ANCOVA models were fit. A Forest plot by subgroups was generated showing least-squares mean estimates and 95\% confidence intervals (CIs) for pairwise differences between paliperidone ER and placebo at week 6 last-observationcarried-forward (LOCF) endpoint. Change from baseline in efficacy measures (observed case) was also analyzed using a repeated-measures mixed-effects linear model. This model included baseline PANSS total score as a covariate; treatment, protocol, country-within-protocol, concomitant medication stratum, and time (scheduled assessment visits on day 4 and at weeks 1, 2, 3, 4, and 6) as factors; and the interaction between time and treatment. The correlation of the repeated measures is modeled with an unstructured covariance matrix. Between-group differences in percentage of subjects who met the composite response were evaluated using Fisher's exact test. Effect sizes were calculated using Cohen's d methodology. Relative risks (RRs) and their corresponding 95\% CIs were calculated for adverse events with $\geq 5 \%$ incidence for paliperidone ER or placebo. RR analyses were considered potentially significant $(P=0.05)$ when the $95 \%$ CIs did not include 1 . No adjustments were made for multiplicity.

\section{Population characteristics}

A total of 627 randomized subjects were in the pooled analysis (Figure 1). In the intent-to-treat analysis set $(n=614), 414$ subjects received paliperidone ER and 200 




Figure I Study flow diagram.

Abbreviation: ER, extended-release.

received placebo. The mean (standard deviation [SD]) age of the combined population was 37.4 (9.9) years (Table 1). In contrast with the male-to-female ratio of patients with schizoaffective disorder in the literature, but typical of the ratio among subjects included in clinical studies, there were more male than female subjects. The majority $(90.9 \%)$ of subjects had a chart diagnosis of schizoaffective disorder prior to screening, and more subjects were diagnosed with the bipolar type than the depressive type. The mean age at first schizoaffective disorder diagnosis was approximately six years later than the age at first psychiatric diagnosis, which was consistent with other observations that the diagnosis emerges over time. ${ }^{43}$ Subjects had received various other previous diagnoses, with schizophrenia being the most common. Nearly one third of subjects had attempted suicide; one half of this group of subjects had made two or more suicide attempts. Mean baseline scores for the PANSS total, YMRS, and HAM-D21 scales indicated that subjects had significant symptoms. Two hundred and seventy-five subjects (45\%) were receiving adjunctive mood stabilizers and/or antidepressants at baseline and continued these medications during the study; $69 \%$ were taking a mood stabilizer, and $49 \%$ were taking an antidepressant. Valproic acid was the most frequently used mood stabilizer; the most frequently used antidepressants were escitalopram, sertraline, and venlafaxine (Table 2). Also, $14 \%$ of subjects in the total population were using medications for extrapyramidal symptoms, and 50\% were using nonbenzodiazepine hypnotics and anxiolytics.

The studies were designed to include dosages from 3 to $12 \mathrm{mg} /$ day, based on the recommended dose range for schizophrenia. ${ }^{31}$ The mean (SD) modal dosage of paliperidone ER in the combined studies was $8.6(2.8) \mathrm{mg}$ /day. Sixty-seven percent of subjects receiving paliperidone ER and $57 \%$ of those receiving placebo completed the study. The most common cause for discontinuation was lack of efficacy for $10 \%$ of the paliperidone ER subjects and $19 \%$ for the placebo subjects (Figure 1).

\section{Efficacy}

The PANSS total score improved significantly more with paliperidone ER than with placebo from day 4 through to 
Table I Baseline demographics and clinical characteristics in two studies of paliperidone ER for schizoaffective disorder (intent-to-treat analysis set)

\begin{tabular}{|c|c|c|c|}
\hline Parameter & Paliperidone ER $(n=4 \mid 4)$ & Placebo $(n=200)$ & Overall $(n=614)$ \\
\hline Age, $y$, mean (SD) & $37.5(9.7)$ & $37.2(10.3)$ & $37.4(9.9)$ \\
\hline \multicolumn{4}{|l|}{ Sex, n (\%) } \\
\hline Male & $250(60.4)$ & $121(60.5)$ & $371(60.4)$ \\
\hline Female & $164(39.6)$ & $79(39.5)$ & $243(39.6)$ \\
\hline \multicolumn{4}{|l|}{ Race, n (\%) } \\
\hline Caucasian & $200(48.3)$ & $100(50.0)$ & $300(48.9)$ \\
\hline African American & $81(19.6)$ & $37(18.5)$ & $118(19.2)$ \\
\hline Asian & $129(31.2)$ & $63(31.5)$ & $192(31.3)$ \\
\hline Other & $4(1.0)$ & $0(0)$ & $4(1.0)$ \\
\hline BMI, $\mathrm{kg} / \mathrm{m}^{2}$, mean (SD) & $27.2(7.3)$ & $27.9(7.6)$ & $27.5(7.4)$ \\
\hline Baseline mood stabilizers or antidepressants, $\mathrm{n}(\%)$ & $186(44.9)$ & $89(44.5)$ & $275(44.8)$ \\
\hline \multirow[t]{2}{*}{ Age at first psychiatric diagnosis, $y$, mean (SD) } & $25.1(8.9)$ & $25.4(10.1)$ & $25.2(9.3)$ \\
\hline & $n=408$ & $n=195$ & $n=603$ \\
\hline $\begin{array}{l}\text { Age at first SCA diagnosis, } y, \\
\text { mean (SD) }\end{array}$ & $31.5(10.1)$ & $32.0(10.4)$ & $31.7(10.2)$ \\
\hline \multicolumn{4}{|l|}{ Prior diagnoses, $\mathrm{n}(\%)^{\mathrm{a}}$} \\
\hline Schizophrenia & $194(46.9)$ & $96(48.0)$ & $290(47.2)$ \\
\hline Bipolar disorder & $119(28.7)$ & $71(35.5)$ & $190(30.9)$ \\
\hline Depression & $72(17.4)$ & $40(20.0)$ & $112(18.2)$ \\
\hline SCA diagnosis prior to screening, & $n=408$ & $n=195$ & $n=603$ \\
\hline $\mathrm{n}(\%)$ & $366(89.7)$ & $182(93.3)$ & $548(90.9)$ \\
\hline SCA disorder type, n (\%) & $n=4 I I$ & $n=200$ & $n=6 I I$ \\
\hline Bipolar & $288(70.0)$ & $133(67.0)$ & $421(68.9)$ \\
\hline \multirow[t]{2}{*}{ Depressive } & $123(30.0)$ & $67(34.0)$ & $190(31.1)$ \\
\hline & $n=409$ & $n=196$ & $n=605$ \\
\hline \multirow{2}{*}{$\begin{array}{l}\text { Total psychiatric hospitalizations, } \\
\text { mean (SD) }\end{array}$} & $6.2(7.6)$ & $6.6(9.9)$ & $6.3(8.4)$ \\
\hline & $n=413$ & $n=199$ & $n=612$ \\
\hline \multicolumn{4}{|l|}{ Suicide attempts, } \\
\hline n (\%) & $128(31.0)$ & $64(32.2)$ & $192(31.4)$ \\
\hline 1 & $68(53.1)$ & $32(50.0)$ & $100(52.1)$ \\
\hline$\geq 2$ & $60(46.9)$ & $32(50.0)$ & $92(47.9)$ \\
\hline Current tobacco use, yes, $n(\%)$ & $240(58.0)$ & $115(57.5)$ & $355(57.8)$ \\
\hline History of alcohol/drug use, yes, $n$ (\%) & $124(30.0)$ & $59(29.5)$ & $183(29.8)$ \\
\hline Alcohol & $79(63.7)$ & $42(71.2)$ & $121(66.1)$ \\
\hline Cocaine & $56(45.2)$ & $30(50.9)$ & $86(47.0)$ \\
\hline Marijuana & $63(50.8)$ & $25(42.4)$ & $88(48.1)$ \\
\hline Other $^{\mathrm{b}}$ & $26(21.0)$ & $10(17.0)$ & $36(19.7)$ \\
\hline \multicolumn{4}{|l|}{ PANSS score, mean (SD) } \\
\hline Total & $93.3(13.2)$ & $91.6(12.3)$ & $92.8(13.0)$ \\
\hline Positive symptoms & $27.4(5.3)$ & $27.1(5.0)$ & $27.3(5.2)$ \\
\hline Negative symptoms & $18.8(6.2)$ & $18.2(5.9)$ & $18.6(6.1)$ \\
\hline Anxiety/depression & $13.2(3.7)$ & $12.9(3.9)$ & $13.1(3.7)$ \\
\hline Disorganized thoughts & $19.7(4.2)$ & $19.3(4.0)$ & $19.6(4.1)$ \\
\hline Hostility/uncontrolled excitement & $14.2(3.0)$ & I4.I (3.I) & $14.2(3.0)$ \\
\hline \multicolumn{4}{|l|}{ CGI-S-SCA, mean (SD) } \\
\hline Total score & $4.6(0.6)$ & $4.6(0.6)$ & $4.6(0.6)$ \\
\hline Positive domain & $4.5(0.7)$ & $4.5(0.7)$ & $4.5(0.7)$ \\
\hline Negative domain & $3.3(1.1)$ & $3.2(1.1)$ & $3.2(1.1)$ \\
\hline Depressive domain & $3.2(1.5)$ & $3.2(1.5)$ & $3.2(1.5)$ \\
\hline Manic domain & $3.5(1.5)$ & $3.5(1.6)$ & $3.5(1.5)$ \\
\hline YMRS, mean (SD) & $24.6(10.1)$ & $24.2(10.1)$ & $24.4(10.0)$ \\
\hline HAM-D-2I, mean (SD) & $20.4(9.0)$ & $19.6(8.3)$ & $20.1(8.8)$ \\
\hline YMRS $\geq 16, n(\%)$ & $328(79.2)$ & $160(80.0)$ & $488(79.5)$ \\
\hline HAM-D-2I $\geq 16, \mathrm{n}(\%)$ & $282(68.1)$ & $129(64.5)$ & $4 I I(66.9)$ \\
\hline YMRS and HAM-D-2I $\geq 16, \mathrm{n}(\%)$ & $196(47.3)$ & $89(44.5)$ & $285(46.4)$ \\
\hline
\end{tabular}

Notes: a Data are not mutually exclusive; ' ${ }^{\circ}$ ther includes heroin, depressants, mixed substance use, and other compounds.

Abbreviations: BMI, body mass index; CGI-S-SCA, Clinical Global Impressions of Severity for Schizoaffective Disorder; ER, extended-release; HAM-D-2I, 2 I-item Hamilton Rating Scale for Depression; PANSS, Positive and Negative Syndrome Scale; SCA, schizoaffective disorder; SD, standard deviation;YMRS, Young Mania Rating Scale; y, years. 
Table 2 Specific medications used by subjects in the adjunctive mood stabilizers and/or antidepressants group $(n=275)$

\begin{tabular}{ll}
\hline Medications & $\mathbf{n}(\%)$ \\
\hline Mood stabilizers & $191(69)$ \\
Valproic acid & $140(5 \mathrm{I})$ \\
Lithium & $43(16)$ \\
Lamotrigine & $6(2)$ \\
Antidepressants & $135(49)$ \\
Escitalopram & $26(9)$ \\
Sertraline & $22(8)$ \\
Venlafaxine & $18(7)$ \\
Fluoxetine & $16(6)$ \\
Trazodone & $16(6)$ \\
Bupropion & $13(5)$ \\
Amitriptyline & $8(3)$ \\
Mirtazapine & $7(3)$ \\
Paroxetine & $7(3)$ \\
Duloxetine & $6(2)$ \\
Citalopram & $5(2)$ \\
Other & $11(4)$ \\
\hline
\end{tabular}

Note: aOther includes tianeptine, imipramine, clomipramine, dosulepin, fluvoxamine, and mianserin.

the study endpoint: placebo-adjusted least-squares mean (standard error) difference at LOCF endpoint (paliperidone ER minus placebo), -7.8 (1.7); $P<0.001$ (Figures 2 and 3). The effect size was 0.41 . Improvements on other measures, including the PANSS factor scores and CGI-S-SCA total and domain scores, were greater with paliperidone ER than with placebo (Table 3). Results using a repeated-measures linear model were consistent with those of the LOCF analysis (Table 3). Composite response rates were higher with paliperidone ER (50\%) than with placebo $(35 \%, P<0.001$, Figure 4).

\section{Paliperidone ER alone or adjunctive to mood stabilizers and/or antidepressants}

Approximately one half of the population was receiving mood stabilizers and/or antidepressants at baseline and continued these medications during the study, which allowed examination of the efficacy of paliperidone ER with or without mood stabilizers and/or antidepressants. Mean (SD) PANSS total scores at baseline were similar for subjects in both groups at 93.1 (13.0) for subjects receiving paliperidone ER as monotherapy and 93.5 (13.6) in those receiving paliperidone ER adjunctive to mood stabilizers and/or antidepressants. Improvement at the six-week endpoint was greater with paliperidone ER than with placebo in both groups (Figure 3).



Figure 2 Mean PANSS total score over time (intent-to-treat analysis set).

Notes: Analysis is based on an ANCOVA model with fixed effects for treatment, study, country nested within study, and the baseline value as a covariate. ap $<0.05$, paliperidone ER versus placebo; ${ }^{b} P<0.01$, paliperidone ER versus placebo.

Abbreviations: ANCOVA, analysis-of-covariance; BL, baseline; ER, extended-release; LOCF, last-observation-carried-forward; PANSS, Positive and Negative Syndrome Scale; SE, standard error 


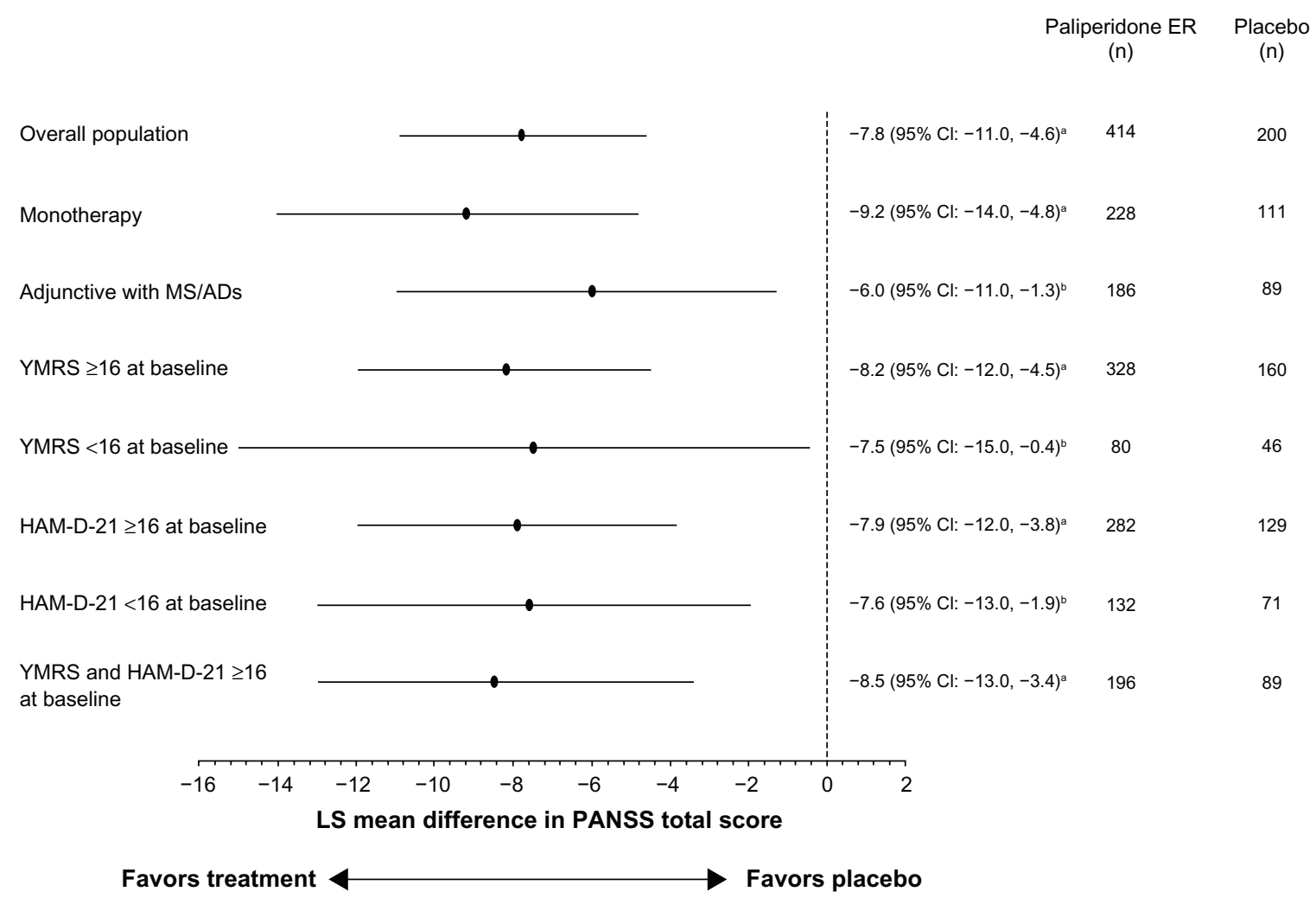

Figure 3 Adjusted mean differences and $95 \%$ Cls for PANSS total change scores at endpoint with paliperidone ER versus placebo (intent-to-treat analysis set). Notes: Analysis is based on an ANCOVA model with fixed effects for treatment, study, country nested within study, and the baseline value as a covariate. ap $\leq 0.00$ I, paliperidone $E R$ versus placebo; ${ }^{b} P<0.05$, paliperidone $E R$ versus placebo.

Abbreviations: Cl, confidence interval; ER, extended-release; HAM-D-2I, 2I-item Hamilton Rating Scale for Depression; LS, least-squares; MS/ADs, mood stabilizers/ antidepressants; PANSS, Positive and Negative Syndrome Scale; YMRS, Young Mania Rating Scale.

Table 3 Efficacy results for the pooled population $(n=614)^{\mathrm{a}}$

\begin{tabular}{|c|c|c|c|c|}
\hline \multirow{2}{*}{$\begin{array}{l}\text { Parameter, change } \\
\text { from baseline }\end{array}$} & \multicolumn{2}{|c|}{ Week 6 (LOCF) endpoint, ANCOVA } & \multicolumn{2}{|c|}{ Week 6 repeated-measures, ANCOVA } \\
\hline & Paliperidone ER & Placebo & Paliperidone ER & Placebo \\
\hline PANSS scores, LS mean (SE) & $n=414$ & $n=200$ & $n=278$ & $n=115$ \\
\hline Total & $-26.2(1.1)^{b}$ & $-18.3(1.5)$ & $-28.5(I .1)^{b}$ & $-21.5(1.6)$ \\
\hline Positive factor & $-8.0(0.4)^{b}$ & $-5.7(0.5)$ & $-8.8(0.4)^{c}$ & $-6.7(0.5)$ \\
\hline Negative factor & $-4.0(0.3)^{b}$ & $-2.6(0.4)$ & $-4.3(0.3)^{d}$ & $-3.2(0.4)$ \\
\hline Anxiety/depression & $-4.2(0.2)^{\mathrm{c}}$ & $-3.3(0.3)$ & $-4.5(0.2)^{d}$ & $-3.8(0.3)$ \\
\hline Disorganized thoughts & $-4.6(0.3)^{b}$ & $-3.0(0.3)$ & $-5.2(0.3)^{c}$ & $-3.8(0.4)$ \\
\hline Uncontrolled hostility/excitement & $-5.4(0.2)^{b}$ & $-4.0(0.3)$ & $-6.1(0.2)^{b}$ & $-4.7(0.3)$ \\
\hline CGI-S-SCA, LS mean (SE) & $n=413$ & $n=200$ & $n=277$ & $n=115$ \\
\hline Overall & $-1.5(0.1)^{\mathrm{b}}$ & $-1.0(0.1)$ & $-1.7(0.1)^{c}$ & $-1.3(0.1)$ \\
\hline Positive domain & $-1.5(0.1)^{\mathrm{b}}$ & $-1.0(0.1)$ & $-1.8(0.1)^{c}$ & $-1.4(0.1)$ \\
\hline Negative domain & $-0.7(0.1)^{c}$ & $-0.5(0.1)$ & $-0.8(0.1)$ & $-0.6(0.1)$ \\
\hline Depressive domain & $-0.9(0.1)^{b}$ & $-0.6(0.1)$ & $-1.0(0.1)^{c}$ & $-0.7(0.1)$ \\
\hline Manic domain & $-1.2(0.1)^{b}$ & $-0.9(0.1)$ & $-1.3(0.1)^{\mathrm{d}}$ & $-1.0(0.1)$ \\
\hline Subjects with YMRS $\geq 16$ & $n=328$ & $n=160$ & $n=219$ & $n=93$ \\
\hline YMRS total, LS mean (SE) & $-14.6(0.7)^{b}$ & $-9.8(0.9)$ & $-16.1(0.7)^{b}$ & $-11.0(1.0)$ \\
\hline Subjects with HAM-D-2I $\geq 16$ & $n=282$ & $n=129$ & $n=178$ & $n=70$ \\
\hline HAM-D-2I total, LS mean (SE) & $-12.7(0.7)^{\mathrm{b}}$ & $-8.9(0.9)$ & $-14.0(0.6)^{c}$ & $-10.9(0.9)$ \\
\hline
\end{tabular}

Notes: The repeated-measures mixed-effects ANCOVA model included the baseline score as a fixed-effect covariate; treatment, concomitant medication stratum, country, study, country nested within study, and time (scheduled visit assessment) as fixed-effect (categoric) factors; and treatment by visit interaction. The correlation of the repeated measures is modeled with an unstructured covariance matrix. ${ }^{2}$ The LOCF analysis is based on an ANCOVA model with fixed effects for treatment, study, country nested within study, and the baseline value as a covariate; ${ }^{b} P \leq 0.00$ I, paliperidone $E R$ versus placebo; ${ }^{c} P<0.0$ I, paliperidone $E R$ versus placebo; ${ }^{d} P<0.05$, paliperidone ER vs placebo.

Abbreviations: ANCOVA, analysis-of-covariance; CGI-S-SCA, Clinical Global Impressions-Severity-Schizoaffective Disorder; ER, extended-release; LS, least-squares; HAM-D-2I, 2I-item Hamilton Rating Scale for Depression; LOCF, last-observation-carried-forward; PANSS, Positive and Negative Syndrome Scale; SE, standard error; YMRS, Young Mania Rating Scale. 


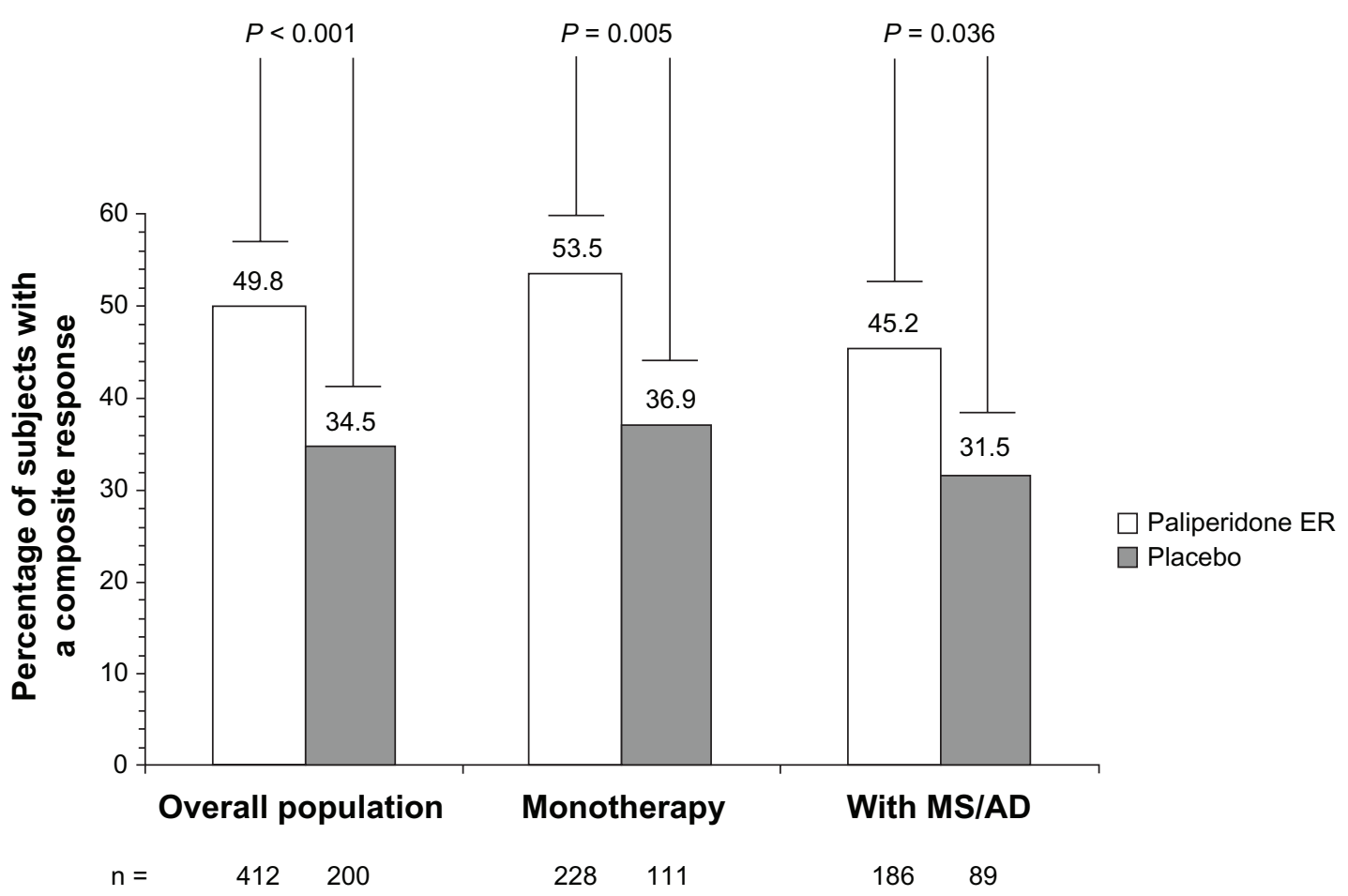

Figure 4 Composite clinical response rate ${ }^{\mathrm{a}}$ at endpoint in the overall population and by concomitant medication stratum (intent-to-treat analysis set).

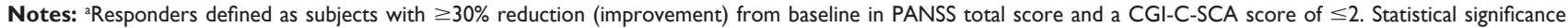
determined using Fisher's Exact test.

Abbreviations: ER, extended-release; CGI-C-SCA, Clinical Global Impressions of Change for Schizoaffective Disorder; MS/AD, mood stabilizer/antidepressant.

\section{Subjects with prominent manic, depressive, or mixed symptoms}

The size of the pooled population allowed for additional subgroup analyses based on the prominence of baseline affective symptoms (subjects with YMRS and/or HAMD-21 score $\geq 16$ at baseline). PANSS total scores improved significantly more with paliperidone ER than with placebo in each of these groups at endpoint (Figure 3).

Further analyses of these subgroups examined the effect of paliperidone ER on mood symptoms. Improvements in YMRS and HAM-D-21 were greater with paliperidone ER than with placebo, as measured by placebo-adjusted least-squares mean $(95 \% \mathrm{CI})$ changes from baseline of -4.8 $(-6.8,-2.9)$ and $-3.8(-5.5,-2.0)$, respectively. Both YMRS and HAM-D-21 scores improved with paliperidone ER as monotherapy or as adjunctive therapy with mood stabilizers and/or antidepressants (Figure 5).

\section{Tolerability}

The most frequent adverse events ( $\geq 5.0 \%$ in paliperidone ER subjects) among subjects receiving paliperidone ER versus placebo were headache (14.3\% versus $14.9 \%)$, tremor $(8.1 \%$ versus $3.5 \%)$, dizziness $(6.7 \%$ versus $5.9 \%)$, insomnia $(6.7 \%$ versus $6.9 \%)$, nausea $(6.4 \%$ versus $5.9 \%)$, akathisia $(5.5 \%$ versus $4.5 \%$ ), hypertonia ( $5.5 \%$ versus $2.0 \%$ ), dyspepsia (5.5\% versus $2.5 \%)$, somnolence $(5.2 \%$ versus $2.0 \%)$, and sedation $(5.0 \%$ versus $3.5 \%)$. An analysis of RR found a greater risk of tremor with paliperidone ER than with placebo $(R R=2.34 ; 95 \% C I=1.05,5.18$, Figure 6). Significance for increased risk (defined as the $95 \%$ CI not including 1) of the other adverse events was not observed.

The overall adverse event rate was higher in subjects who received paliperidone ER as adjunctive therapy to mood stabilizers and/or antidepressants (70.4\%, paliperidone ER; $70.0 \%$, placebo) than in those who received paliperidone ER as monotherapy (60.5\%, paliperidone ER; $49.1 \%$, placebo). An analysis of RR found a greater risk of tremor with paliperidone $\mathrm{ER}$ than with placebo $(\mathrm{RR}=4.38 ; 95 \% \mathrm{CI}=1.04$, 18.56) in the monotherapy group (Figure 6). Significance for increased risk of adverse events was not observed in the mood stabilizer and/or antidepressant group.

The percentage of discontinuations due to adverse events was similar for both the paliperidone ER- and the placebotreated groups (each 7\%) but was slightly higher in the group receiving paliperidone ER adjunctive to mood stabilizers and/or antidepressants $(8.2 \%)$ than in the group receiving paliperidone ER as monotherapy (5.0\%). 


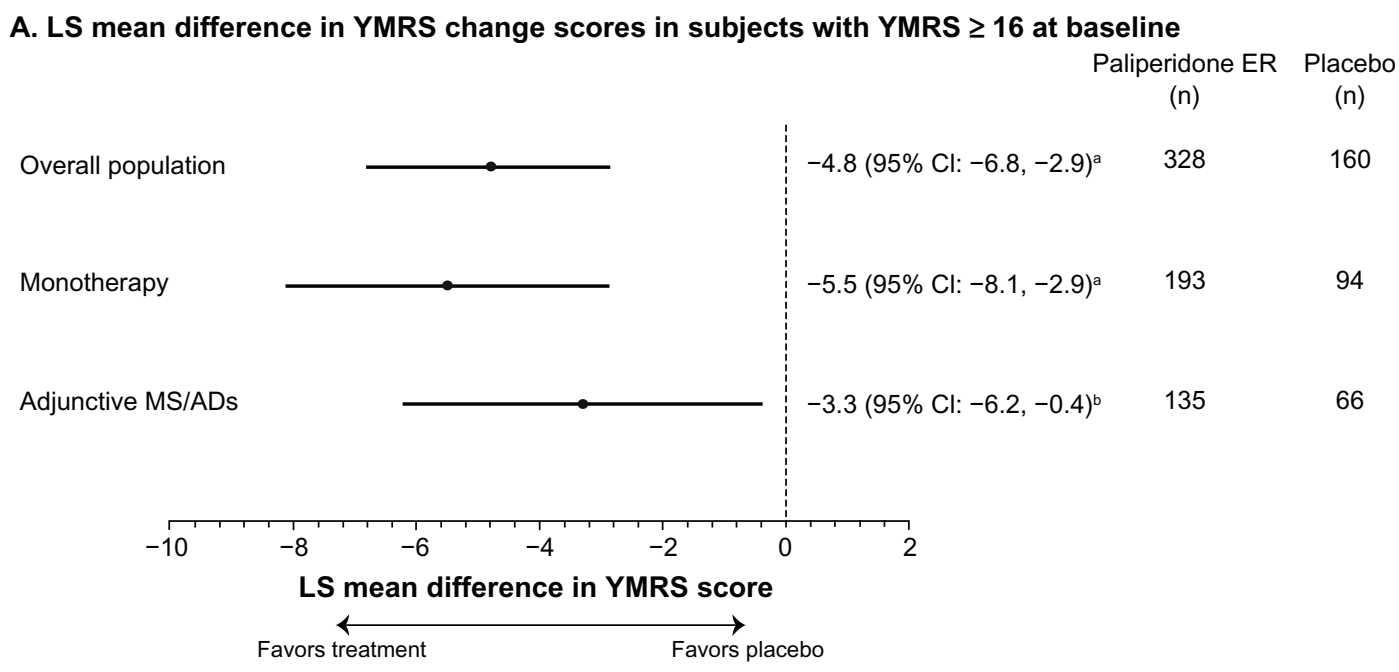

B. LS mean difference in HAM-D-21 change scores in subjects with HAM-D-21 $\geq 16$ at baseline

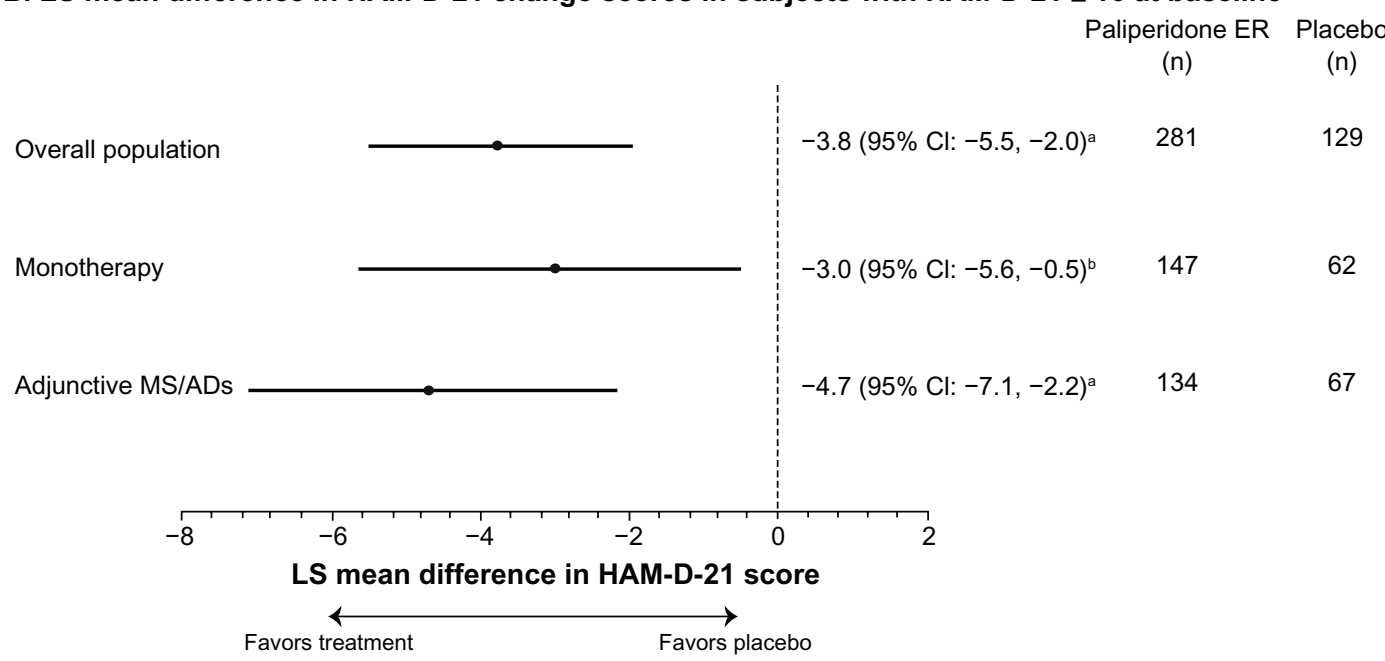

Figure 5 Adjusted mean differences and 95\% confidence intervals for total YMRS and HAM-D-2I change scores at endpoint with paliperidone ER versus placebo in patients with manic or depressive symptoms at baseline (intent-to-treat analysis set).

Notes: Analysis is based on an ANCOVA model with fixed effects for treatment, study, country nested within study, and the baseline value as a covariate. ${ }^{2} \leq \leq 0.00$, paliperidone $E R$ versus placebo; ${ }^{b} P<0.05$, paliperidone $E R$ versus placebo.

Abbreviations: Cl, confidence interval; ER, extended-release; HAM-D-2I, 2 I-item Hamilton Rating Scale for Depression; LS, least-squares; MS/ADs, mood stabilizers/ antidepressants; PANSS, Positive and Negative Syndrome Scale; YMRS, Young Mania Rating Scale.

Adverse events of particular relevance with antipsychotic therapy are extrapyramidal symptoms, weight and metabolic changes, and prolactin elevation. Extrapyramidal symptoms were reported in $19.8 \%$ of subjects receiving paliperidone ER and in $10.9 \%$ of those receiving placebo. The severity of extrapyramidal symptoms as measured using the SAS, AIMS, and BAS was low (global score $<1$ ) in both groups at baseline and endpoint.

At endpoint, mean (SD) changes in prolactin levels $(\mathrm{ng} / \mathrm{mL})$ were greater with paliperidone ER versus placebo for both males (12.6 [20.1] versus -3.6 [13.3]) and females (51.4 [62.1] versus -13.9 [32.4]). There were 9 $(2.1 \%)$ subjects receiving paliperidone ER and one $(0.5 \%)$ subject receiving placebo who experienced a potentially prolactin-related adverse event. The mean (SD) weight change was 1.0 (2.7) $\mathrm{kg}$ for paliperidone ER and $0.2(2.1) \mathrm{kg}$ for placebo. There were no clinically relevant differences in the lipid profile or fasting glucose levels for paliperidone ER compared with placebo.

Additionally, no clinically relevant differences were found in the incidence of extrapyramidal symptoms or in changes in mean weight, lipid profile, fasting glucose level, or prolactin level between subjects who received paliperidone ER as monotherapy and those who received paliperidone ER adjunctive to mood stabilizers and/or antidepressants (Table 4). 


\section{A. Overall population}

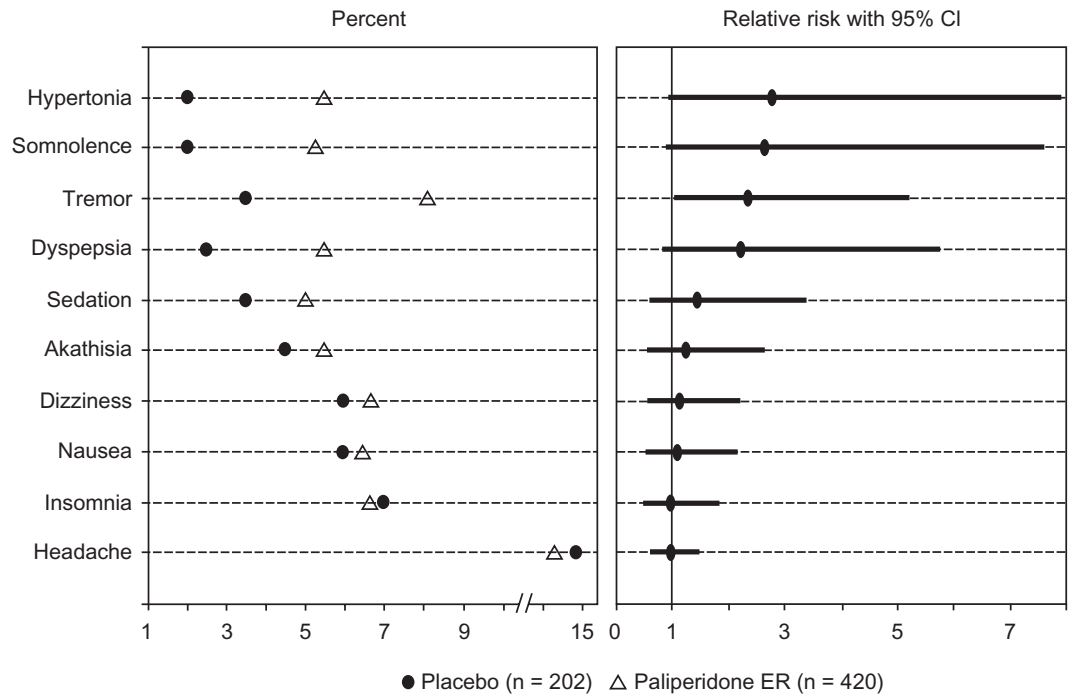

B. Monotherapy

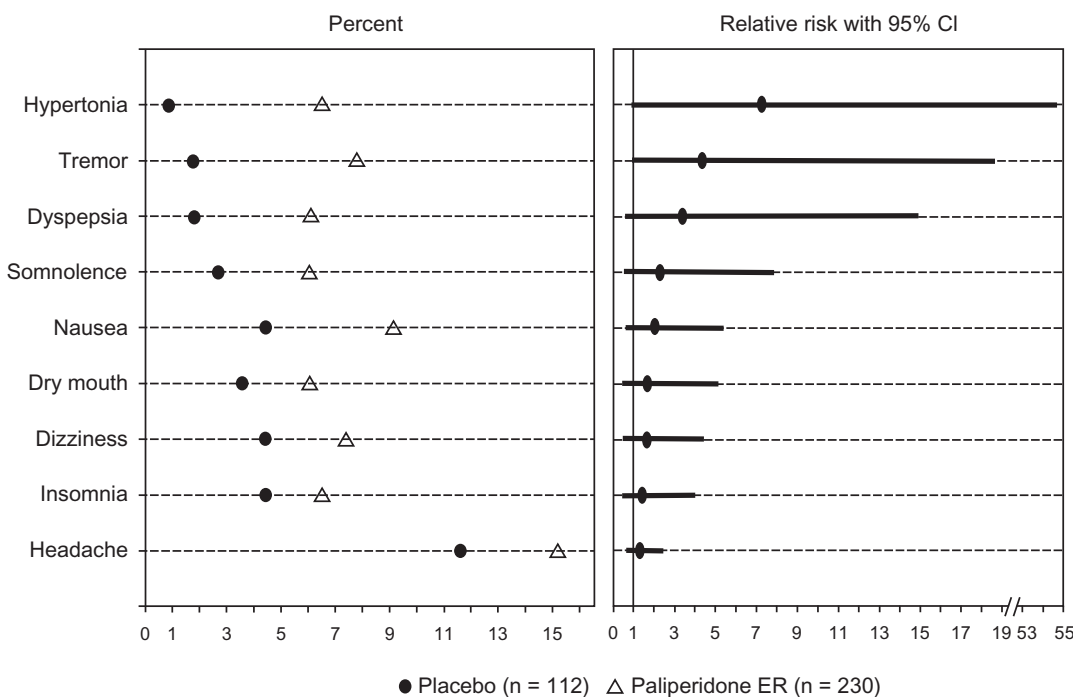

\section{Adjunctive MS/ADs}

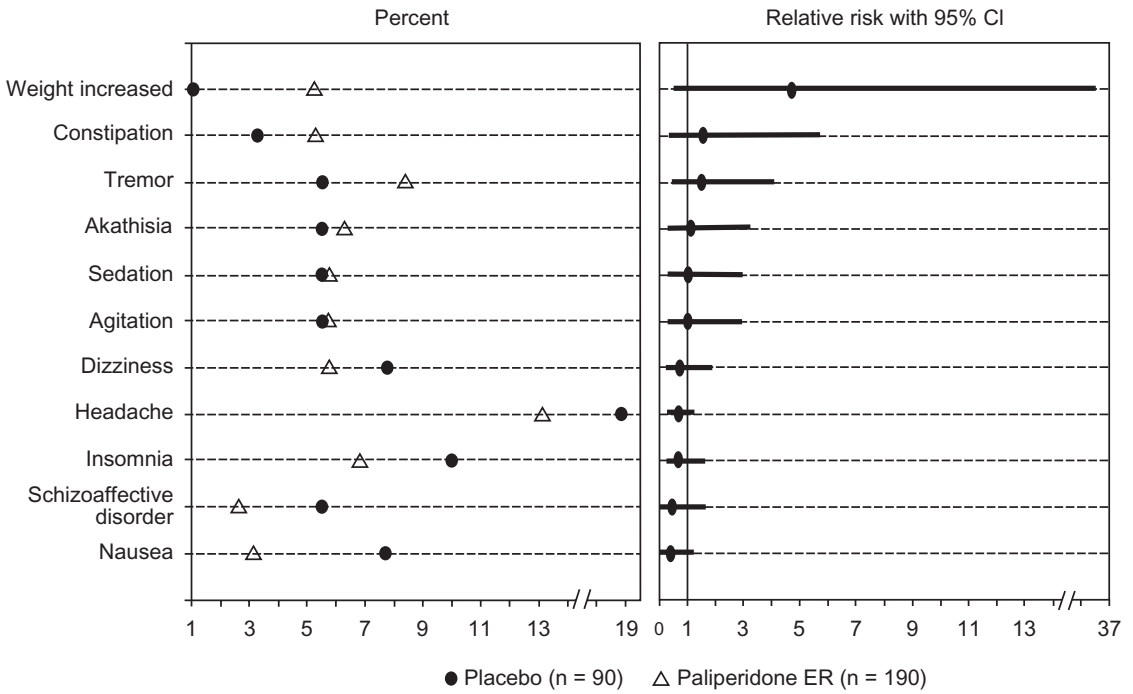

Figure 6 Most frequent (in $\geq 5.0 \%$ of paliperidone ER subjects) adverse events sorted by relative risk (safety analysis set) by concomitant medication stratum. Abbreviations: $\mathrm{Cl}$, confidence interval; $\mathrm{ER}$, extended-release; MS/AD, mood stabilizer/antidepressant. 


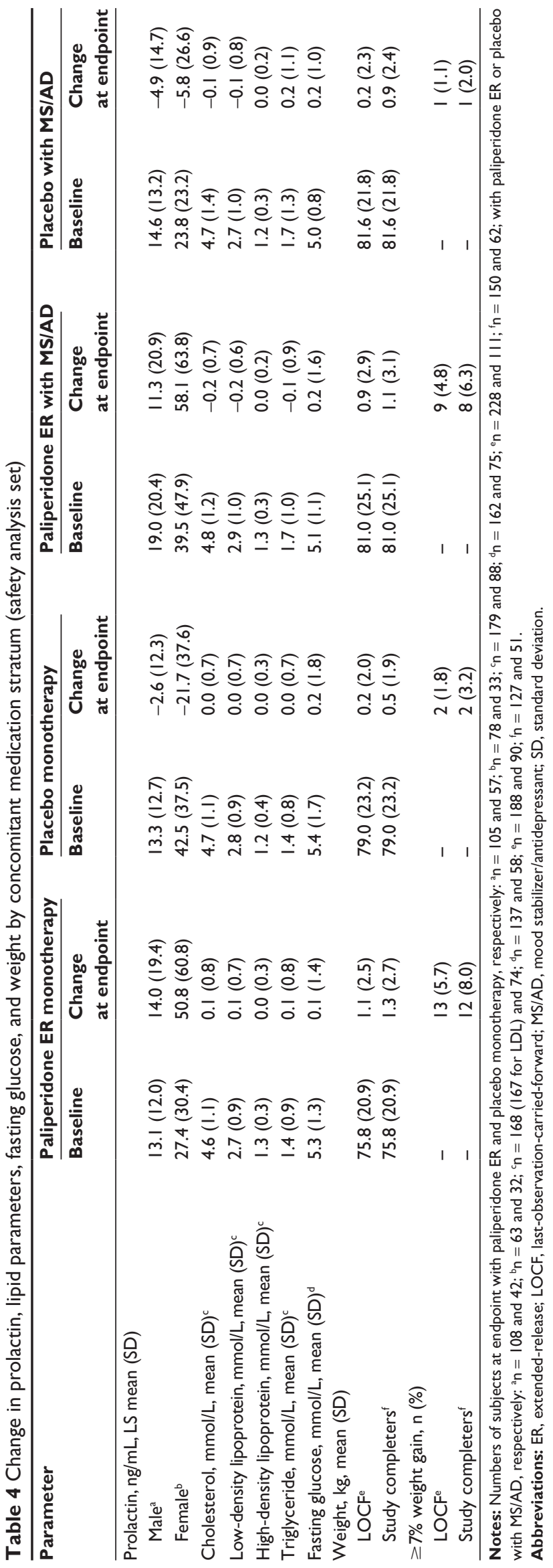

\section{Discussion}

To our awareness, this review covers the largest data set of patients with schizoaffective disorder, and provides additional knowledge of patient characteristics and treatment responses to paliperidone ER. Although differential diagnosis across the psychosis and mood continuum can be challenging, over $90 \%$ of subjects carried a chart diagnosis of schizoaffective disorder prior to enrollment in the studies. Interestingly, the mean age at first schizoaffective disorder diagnosis was approximately six years later than the age at first psychiatric diagnosis. This is consistent with the longitudinal nature of the diagnosis, which requires assessment of the duration of mood symptoms relative to the total duration of illness, and suggests that full symptom expression emerges over time. All subjects' diagnoses were confirmed by the Structured Clinical Interview for DSM-IV Disorders at study entry. Their mean age was approximately 37 years, with an average of six psychiatric hospitalizations per subject, and a previous suicide attempt reported in over $30 \%$ of subjects. These subjects were acutely ill at study entry and displayed high levels of concurrent psychotic, manic, and depressive symptoms. Notably, approximately one half of this population was receiving concomitant mood stabilizers and/or antidepressants at baseline.

The data presented here demonstrate that patients with schizoaffective disorder can be successfully treated with paliperidone ER. Although the mean modal dosage of paliperidone ER in these data was $8.6 \mathrm{mg} / \mathrm{day}$, the recommended dosage for schizoaffective disorder is $6 \mathrm{mg} /$ day, with a range of 3 to $12 \mathrm{mg} /$ day. The dosing recommendation is supported by the study designs that utilize this range, and the majority of subjects randomized to paliperidone ER were initiated at $6 \mathrm{mg}$ /day. Additionally, although the lower-dosage group (3 to $6 \mathrm{mg}$ /day) in the two-dose group study was not superior to placebo with regard to PANSS total change, it was associated with a significantly higher composite response rate. ${ }^{29}$

Treatment-emergent adverse events (including extrapyramidal symptom-related adverse events) and changes in weight and metabolic parameters that were identified in these studies were similar to those found in studies of paliperidone ER in schizophrenia. ${ }^{44}$ As expected, the potent $\mathrm{D}_{2}$ antagonism of paliperidone ER compared with other atypical antipsychotics resulted in an elevation in serum prolactin levels, with females having a higher increase than males. Of note, a previously reported study found that use of paliperidone ER resulted in increases in prolactin levels similar to those found with the use of risperidone..$^{45}$

Because patients with schizoaffective disorder are commonly treated with antipsychotics and mood stabilizers and/or 
antidepressants, ${ }^{26-28}$ results suggesting that paliperidone ER is efficacious both as monotherapy and adjunctive to mood stabilizers and/or antidepressants may be particularly valuable to the clinician. With the exception of tremor, the tolerability of paliperidone ER was comparable when used as monotherapy or adjunctive to mood stabilizers and/or antidepressants. However, it must be noted that although randomization was stratified by mood stabilizer and/or antidepressant use, the study was not designed to compare monotherapy with adjunctive therapy or to compare the relative efficacy of various adjunctive combinations, and the benefits and risks of specific combinations of paliperidone ER and mood stabilizers and/or antidepressants have not been studied. Nonetheless, no unique tolerability issues were identified with paliperidone ER in this population. Because of its limited liability for hepatically mediated drug-drug interactions, paliperidone ER may be a useful option for patients who follow complex medication regimens. ${ }^{32-34}$

Several additional factors must be considered in the interpretation of these results. First, this study population may have been biased toward subjects experiencing manic symptoms because of the specified entry criteria. Nevertheless, in addition to the $80 \%$ of subjects who displayed prominent manic symptoms, $67 \%$ of subjects displayed prominent depressive symptoms at baseline. Moreover, subjects with prominent manic, depressive, or mixed symptoms at baseline had significant psychotic and mood symptom improvement with paliperidone ER compared with placebo. Finally, longterm maintenance treatment with paliperidone ER has not yet been studied in this population. However, such a trial is planned with paliperidone palmitate, the once-monthly injectable formulation of paliperidone.

\section{Conclusion}

Although schizoaffective disorder is commonly encountered in populations of patients with mental illness, this disorder has not been methodically studied, and no established guidelines exist for the management of these patients. Data pooled from two large, international, placebo-controlled, six-week studies with paliperidone ER are now available, demonstrating that acutely ill patients with schizoaffective disorder manifest high levels of psychotic, manic, and depressive symptoms at baseline. Patients who received paliperidone ER, either as monotherapy or adjunctive to mood stabilizers and/or antidepressants, experienced significant improvement in schizoaffective symptoms. Subjects with prominent manic, depressive, or mixed symptoms at baseline showed significant psychotic and mood symptom improvement with paliperidone ER compared with placebo. Additionally, no new tolerability signals were detected in this patient population. These data demonstrate the efficacy and tolerability of paliperidone ER as monotherapy or adjunctive to mood stabilizers and/or antidepressants in the acute treatment of schizoaffective disorder.

\section{Acknowledgment}

The authors wish to acknowledge Mariana Ovnic, Matthew Grzywacz, and ApotheCom (funding supported by OrthoMcNeil Janssen Scientific Affairs, LLC, Titusville, NJ) for technical assistance in the development and submission of this manuscript.

\section{Disclosure}

This study was supported by Ortho-McNeil Janssen Scientific Affairs, LLC. CM Canuso and I Turkoz are employees of Johnson \& Johnson Pharmaceutical Research and Development, LLC, and Johnson \& Johnson stockholders. CA Bossie and DJ Fu are employees of Ortho-McNeil Janssen Scientific Affairs, LLC, and Johnson \& Johnson stockholders.

\section{References}

1. Kasanin J. The acute schizoaffective psychoses. 1933. Am J Psychiatry. 1994;151 Suppl 6:144-154.

2. Perala J, Suvisaari J, Saarni SI, et al. Lifetime prevalence of psychotic and bipolar I disorders in a general population. Arch Gen Psychiatry. 2007;64:19-28.

3. Canuso CM, Kosik-Gonzalez C, Sheehan J, et al. Frequency of schizoaffective disorder diagnosis in patients with psychotic disorders using the mini-international neuropsychiatric interview. Schizophr Res. 2010;98:305-306

4. Kent S, Fogarty M, Yellowlees P. Heavy utilization of inpatient and outpatient services in a public mental health service. Psychiatr Serv. 1995;46:1254-1257.

5. Mattes JA, Nayak D. Lithium versus fluphenazine for prophylaxis in mainly schizophrenic schizo-affectives. Biol Psychiatry. 1984; 19:445-449

6. Brockington IF, Kendell RE, Kellett JM, Curry SH, Wainwright S. Trials of lithium, chlorpromazine and amitriptyline in schizoaffective patients. Br J Psychiatry. 1978;133:162-168.

7. Janicak PG, Keck PE Jr, Davis JM, et al. A double-blind, randomized, prospective evaluation of the efficacy and safety of risperidone versus haloperidol in the treatment of schizoaffective disorder. J Clin Psychopharmacol. 2001;21:360-368.

8. Okuma T, Yamashita I, Takahashi R, et al. A double-blind study of adjunctive carbamazepine versus placebo on excited states of schizophrenic and schizoaffective disorders. Acta Psychiatr Scand. 1989;80:250-259.

9. Chengappa KNR, David JK, Haranath P. A placebo-controlled, randomassignment, parallel-group pilot study of adjunctive topiramate for patients with schizoaffective disorder, bipolar type. Bipolar Disord. 2007;9:609-617.

10. Greil W, Ludwig-Mayerhofer W, Erazo N, et al. Lithium vs carbamazepine in the maintenance treatment of schizoaffective disorder: A randomised study. Eur Arch Psychiatry Clin Neurosci. 1997; 247:42-50.

11. Cheniaux E, Landeira-Fernandez J, Lessa TL, et al. Does schizoaffective disorder really exist? A systematic review of the studies that compared schizoaffective disorder with schizophrenia or mood disorders. J Affect Disord. 2008;106:209-217. 
12. Lindenmayer JP, Kay SR, van PH. Distinction of schizoaffective from schizophrenic profile. Independent validation. Schizophr Res. 1989;2:423-424.

13. Olfson M, Marcus SC, Wan GJ. Treatment patterns for schizoaffective disorder and schizophrenia among Medicaid patients. Psychiatr Serv. 2009;60:210-216.

14. Craddock N, Jones L, Jones IR, et al. Strong genetic evidence for a selective influence of GABAA receptors on a component of the bipolar disorder phenotype. Mol Psychiatry. 2010;15:146-153.

15. Hamshere ML, Green EK, Jones IR, et al. Genetic utility of broadly defined bipolar schizoaffective disorder as a diagnostic concept. $\mathrm{BrJ}$ Psychiatry. 2009;195:23-29.

16. Lencz T, Lipsky RH, DeRosse P, Burdick KE, Kane JM, Malhotra AK. Molecular differentiation of schizoaffective disorder from schizophrenia using BDNF haplotypes. Br J Psychiatry. 2009;194:313-318.

17. Malhi GS, Berk M, Bourin M, et al. Atypical mood stabilizers: A "typical" role for atypical antipsychotics. Acta Psychiatr Scand Suppl. 2005;111 Suppl 426:29-38.

18. Derry S, Moore RA. Atypical antipsychotics in bipolar disorder: Systematic review of randomised trials. BMC Psychiatry. 2007;7:40.

19. Cruz N, Sanchez-Moreno J, Torres F, Goikolea JM, Valenti M, Vieta E. Efficacy of modern antipsychotics in placebo-controlled trials in bipolar depression: A meta-analysis. Int J Neuropsychopharmacol. 2010;13:5-14.

20. Keck PE Jr, McElroy SL, Strakowski SM. Schizoaffective disorder: Role of atypical antipsychotics. Schizophr Res. 1999;35 Suppl: S5-S12.

21. Vieta E, Goikolea JM, Corbella B, et al. Risperidone safety and efficacy in the treatment of bipolar and schizoaffective disorders: Results from a 6-month, multicenter, open study. J Clin Psychiatry. 2001 62:818-825.

22. Lasser R, Bossie CA, Gharabawi G, Eerdekens M, Nasrallah HA Efficacy and safety of long-acting risperidone in stable patients with schizoaffective disorder. J Affect Disord. 2004;83:263-275.

23. Tran PV, Tollefson GD, Sanger TM, Lu Y, Berg PH, Beasley CM Jr Olanzapine versus haloperidol in the treatment of schizoaffective disorder. Acute and long-term therapy. Br J Psychiatry. 1999;174:15-22.

24. Keck PE Jr, Reeves KR, Harrigan EP; and the Ziprasidone Study Group. Ziprasidone in the short-term treatment of patients with schizoaffective disorder: Results from two double-blind, placebo-controlled, multicenter studies. J Clin Psychopharmacol. 2001;21:27-35.

25. Glick ID, Mankoski R, Eudicone JM, Marcus RN, Tran QV, AssunçãoTalbott S. The efficacy, safety, and tolerability of aripiprazole for the treatment of schizoaffective disorder: Results from a pooled analysis of a sub-population of subjects from two randomized, double-blind, placebo-controlled, pivotal trials. J Affect Disord. 2009;115:18-26.

26. Levinson DF, Umapathy C, Musthaq M. Treatment of schizoaffective disorder and schizophrenia with mood symptoms. Am J Psychiatry. 1999;156:1138-1148.

27. McElroy SL, Keck PE Jr, Strakowski SM. An overview of the treatment of schizoaffective disorder. J Clin Psychiatry. 1999;60 Suppl 5: $16-21$.

28. Flynn J, Grieger TA, Benedek DM. Pharmacologic treatment of hospitalized patients with schizoaffective disorder. Psychiatr Serv. 2002;53:94-96.
29. Canuso CM, Lindenmayer JP, Kosik-Gonzalez C, et al. A randomized, double-blind, placebo-controlled study of 2 dose ranges of paliperidone extended-release in the treatment of subjects with schizoaffective disorder. J Clin Psychiatry. 2010;71:587-598.

30. Canuso CM, Schooler N, Carothers J, et al. Paliperidone ER in schizoaffective disorder: A randomized controlled study comparing a flexible dose with placebo in patients treated with and without antidepressants and/or mood stabilizers. J Clin Psychopharmacol. 2010;30:487-495.

31. Invega ${ }^{\circledR}$ (paliperidone) extended-release tablets [Prescribing Information]. Titusville, NJ: Janssen, Division of Ortho-McNeil-Janssen Pharmaceuticals, Inc; 2010.

32. Conley RR, Kelly DL. Drug-drug interactions associated with secondgeneration antipsychotics: Considerations for clinicians and patients. Psychopharmacol Bull. 2007;40:77-97.

33. Vermeir M, Naessens I, Remmerie B, et al. Absorption, metabolism, and excretion of paliperidone, a new monoaminergic antagonist, in humans. Drug Metab Dispos. 2008;36:769-779.

34. Berwaerts J, Cleton A, Herben V, et al. The effects of paroxetine on the pharmacokinetics of paliperidone extended-release tablets. Pharmacopsychiatry. 2009;42:158-163.

35. Kay SR, Fiszbein A, Opler LA. The positive and negative syndrome scale (PANSS) for schizophrenia. Schizophr Bull. 1987;13:261-276.

36. Young RC, Biggs JT, Ziegler VE, Meyer DA. A rating scale for mania: Reliability, validity and sensitivity. Br J Psychiatry. 1978;133:429-435.

37. Hamilton M. Development of a rating scale for primary depressive illness. Br J Soc Clin Psychol. 1967;6:278-296.

38. Allen MH, Bartko JJ, Lindenmayer JP, et al. Development and psychometric exploration of a clinical global impressions scale for schizoaffective disorder (CGI-SCA). Poster presented at 49th Annual New Clinical Drug Evaluation Unit Meeting, 2009 Jun 29-Jul 2, Hollywood, FL.

39. Marder SR, Davis JM, Chouinard G. The effects of risperidone on the five dimensions of schizophrenia derived by factor analysis: Combined results of the North American trials. J Clin Psychiatry. 1997 ; 58:538-546.

40. Simpson GM, Angus JW. A rating scale for extrapyramidal side effects. Acta Psychiatr Scand Suppl. 1970;212:11-19.

41. Guy W. Abnormal Involuntary Movement Scale. ECDEU Assessment Manual for Psychopharmacology. Washington, DC: U.S. Public Health Service; 1976.

42. Barnes TR. A rating scale for drug-induced akathisia. Br J Psychiatry. 1989;154:672-676.

43. Salvatore P, Baldessarini RJ, Tohen M, et al. McLean-Harvard international first-episode project: Two-year stability of DSM-IV diagnoses in 500 first-episode psychotic disorder patients. J Clin Psychiatry. 2009;70:458-466.

44. Meltzer HY, Bobo WV, Nuamah IF, et al. Efficacy and tolerability of oral paliperidone extended-release tablets in the treatment of acute schizophrenia: Pooled data from three 6-week, placebo-controlled studies. J Clin Psychiatry. 2008;69:817-829.

45. Berwaerts J, Cleton A, Rossenu S, et al. A comparison of serum prolactin concentrations after administration of paliperidone extended-release and risperidone tablets in patients with schizophrenia. J Psychopharmacol. 2010;24:1011-1018
Neuropsychiatric Disease and Treatment

\section{Publish your work in this journal}

Neuropsychiatric Disease and Treatment is an international, peerreviewed journal of clinical therapeutics and pharmacology focusing on concise rapid reporting of clinical or pre-clinical studies on a range of neuropsychiatric and neurological disorders. This journal is indexed on PubMed Central, the 'PsycINFO' database and CAS, and is the officia

\section{Dovepress}

journal of The International Neuropsychiatric Association (INA). The manuscript management system is completely online and includes a very quick and fair peer-review system, which is all easy to use. Visit http://www.dovepress.com/testimonials.php to read real quotes from published authors. 